\title{
Factors resulting in postoperative dysphagia following esophagectomy: a narrative review
}

\author{
Cecilia Benz ${ }^{1}$, Jessica Martella ${ }^{2}$, Basel Hamwi ${ }^{2}$, Ikenna Okereke ${ }^{3}$ \\ ${ }^{1}$ Division of Cardiothoracic Surgery, University of Texas Medical Branch, Galveston, TX, USA; ${ }^{2}$ University of Texas Medical Branch School of \\ Medicine, Galveston, TX, USA; ${ }^{3}$ Division of Thoracic Surgery, Henry Ford Health, Detroit, MI, USA \\ Contributions: (I) Conception and design: I Okereke, C Benz; (II) Administrative support: I Okereke; (III) Provision of study materials or patients: \\ C Benz, J Martella, B Hamwi; (IV) Collection and assembly of data: C Benz, J Martella, B Hamwi; (V) Data analysis and interpretation: C Benz, J \\ Martella, B Hamwi; (VI) Manuscript writing: All authors; (VII) Final approval of manuscript: All authors. \\ Correspondence to: Ikenna Okereke, MD. Vice Chairman, Department of Surgery; System Director, Thoracic Surgery, Henry Ford Health, Detroit, \\ MI, 48202, USA. Email: ikokerek@utmb.edu.
}

\begin{abstract}
Esophagectomy is a technically involved surgery and can have significant postoperative morbidity. Although the mortality rate following esophagectomy has decreased in recent years, this surgical procedure has a relatively high complication rate compared to other surgeries to resect cancer. One of the most common complaints after esophagectomy is dysphagia. Dysphagia after esophagectomy can significantly affect quality of life. Dysphagia is a complication following esophagectomy that can lead to respiratory deterioration and death. The most common sites of postoperative dysphagia are the gastroesophageal anastomosis, gastric conduit, pylorus and the hiatus. Without appropriate treatment of dysphagia, malnutrition and dehydration can develop. These factors can lead to significant impacts to the overall health of a patient and increase mortality. A detailed literature review provided data to support diagnostic modalities and management strategies to treat postoperative dysphagia at these common areas. A systematic, evidencebased approach to diagnosis and treatment of postoperative dysphagia allows for prompt intervention and a decrease in morbidity and mortality. Treatment options for dysphagia vary, depending on the etiology. Based on the location and mechanism of dysphagia, options include stenting, dilation and surgical revision. Early treatment of dysphagia after esophagectomy can lessen the morbidity from this complication and improve quality of life.
\end{abstract}

Keywords: Esophagectomy; dysphagia; complications

Submitted Apr 24, 2021. Accepted for publication Jun 11, 2021.

doi: 10.21037/jtd-21-724

View this article at: https://dx.doi.org/10.21037/jtd-21-724

\section{Introduction}

Esophagectomy is a common surgical procedure performed for both benign and malignant conditions. It can be approached transthoracically, transabdominally, minimally invasively or open based on the location of the targeted area, availability of conduit, patient comorbidities and surgeon preference.

Although mortality associated with esophagectomy has decreased in recent years, morbidity remains a concern. Perioperative complications include specific complaints related to postoperative surgical anatomy, as well as respiratory, cardiac, and neurologic complications associated with major surgery.

Dysphagia is a common postoperative complaint, with up to 65 percent of esophagectomy patients suffering from some degree of dysphagia. Dysphagia after esophagectomy is managed based on the etiology of symptoms. Dysphagia is a major concern in a population at high risk for malnutrition and requires prompt evaluation and intervention. However, there is a paucity of data for post-esophagectomy patients with dysphagia and recommendations for diagnostic and treatment algorithms (Table 1). 
Table 1 Additional studies for suspected area causing dysphagia

Follow-up study
Gastroesophageal anastomosis
Endoscopy with biopsy
Barium swallow
Gastric conduit
Endoscopy
Gastric motility study
Computed tomography
Pylorus
Endoscopy
Barium swallow
Hiatus
Computed tomography
Endoscopy

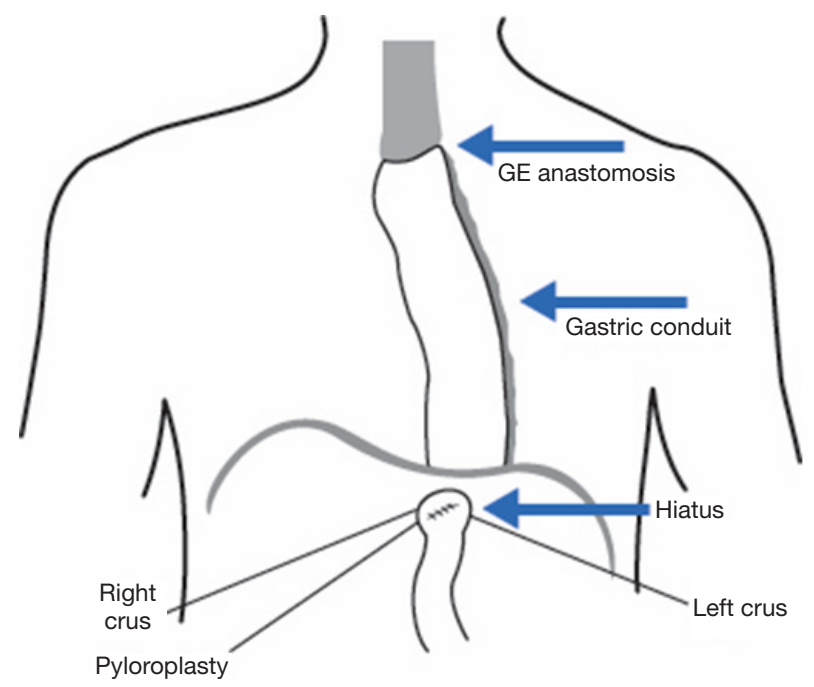

Figure 1 Potential sites causing postoperative dysphagia.

The sites of postoperative dysphagia correlate with surgical changes in native anatomy (Figure 1). When a gastric conduit is used to replace the esophagus during esophagectomy, the gastroesophageal anastomosis is a common etiology for dysphagia secondary to stricture development. Distal to the anastomosis, the gastric conduit can be a source of dysphagia due to anatomic changes in the shape and course, as well as poor peristalsis and functional changes of the remaining stomach. Pyloric dysfunction postoperatively can result in dysphagia, but preemptive operative intervention can lead to problems with gastric emptying. With proper imaging and endoscopic studies, as well as a systematic approach to evaluation of the postoperative anatomy, the etiology of postoperative dysphagia can be promptly diagnosed and appropriately treated.

To perform this literature review, an online electronic search was performed of all literature with the following keywords: esophagectomy, postoperative dysphagia, stricture, functional dysphagia, anastomosis, pyloroplasty, pyloromyotomy, gastric conduit and hiatal hernia.

We present the following article in accordance with the Narrative Review reporting checklist (available at https:// dx.doi.org/10.21037/jtd-21-724).

\section{Gastroesophageal anastomosis}

Dysphagia is a common presenting symptom of anastomotic dysfunction following esophagectomy. Causes of dysfunction can be anatomic or functional. Anastomotic stricture is a common post-esophagectomy complication, occurring in approximately 30 percent of patients following esophagectomy (1). Anastomotic strictures after esophagectomy can be secondary to benign or malignant conditions. The most frequent cause of anastomotic stricture after esophagectomy is from a perioperative anastomotic leak. These leaks usually occur secondary to ischemia or excessive tension on the anastomosis. Newer modalities such as thermography and fluorescence imaging may have a role in identifying ischemic areas of the gastric conduit prior to anastomosis. These studies are ongoing.

An anastomotic stricture which develops remotely after surgery should stimulate a workup to rule out recurrence of cancer. Recurrent cancer at the anastomosis can be treated with dilation or stenting to alleviate symptoms if present. Thereafter, chemotherapy and/or radiation treatment are potential options. Rarely, repeat resection of disease will be possible.

Dysphagia from a stricture should be differentiated from functional dysphagia, as these two situations have very different treatment plans. Functional dysphagia commonly results after subtotal esophagectomy with cervical anastomosis. In these cases, the remaining esophagus is very short and has poor peristaltic ability after a swallow. In addition, the cervical incision, malnutrition and denervation of the gastric conduit can all lead to delayed gastric 
emptying (2).

Generally, a postoperative stricture at the gastroesophageal anastomosis is identified based on symptoms of dysphagia and a sensation of food being "stuck" in the mid-chest or neck area depending on the type of esophagectomy performed. The etiology of the stricture can be determined using barium swallow study, endoscopy, and/or diagnostic imaging (3). Surveillance endoscopy has limited benefit in preventing stricture or mitigating the morbidity from a stricture. Recent studies have shown that cancer recurrence was first suspected based on symptoms in 50 percent of cases, computed tomography (CT) scan in 45 percent of cases and from routine endoscopy in less than 1 percent of cases (3). Most cases of cancer recurrence occur in the first two years after surgery. For this reason, most surveillance strategies utilize CT scan imaging every 6 months for the first 2 years and then yearly afterward (3).

Risk factors for benign stricture include the location of anastomosis, pre-existing comorbidities and a perioperative anastomotic leak. When performing a transhiatal esophagectomy specifically, leak rates are associated with poor preoperative nutritional status, respiratory complications and intraoperative blood loss (4). Retrospective series have shown higher leak rates and subsequent stricture formation in patients with cervical anastomoses (5). The technique used to create the anastomosis, such as stapled versus hand-sewn, does not appear to affect leak or stricture rate. And current data seems to show no correlation between preoperative chemoradiation treatment and stricture formation. (6).

Once a benign stricture is diagnosed, endoscopy and balloon dilation is the usual first therapeutic treatment modality used (7). Although many patients will develop some degree of dysphagia after esophagectomy, only a small fraction of those patients will ever need dilation of their anastomosis (2). Multiple balloon dilations are often required to treat benign anastomotic strictures successfully (8). When dilation is needed, over 50 percent of patients require at least two separate dilations to relieve (1). There is also evidence that some anastomotic strictures can resolve over time without requiring balloon dilation (9). Recent meta-analyses have shown that dilations can improve quality of life in patients with anastomotic strictures, especially when steroid injection is performed during the dilation (10). Benign strictures refractory to dilation may ultimately require temporary esophageal stenting with endoscopy (11).

Balloon dilation under fluoroscopic guidance has reliable success in treating benign anastomotic strictures after esophagectomy. Benign strictures secondary to scar contracture are ideal for balloon dilation. A patient with a stricture secondary to fistula formation, however, may be worsened by dilation if the fistula widens. These varied potential results emphasize the importance of a comprehensive diagnostic workup and a multidisciplinary approach (2). Endoscopy and diagnostic imaging should be used to determine the etiology of a stricture prior to attempting balloon dilation.

While most early gastroesophageal anastomotic strictures are benign, late anastomotic stricture are usually malignant. A patient who develops new onset of an anastomotic stricture more than 12 months after esophagectomy should be evaluated for local cancer recurrence. These strictures require tissue biopsy during endoscopy, and diagnosis of local recurrence is associated with a poor prognosis. Risk factors include surgical margins less than 5 centimeters and nodal status. Following diagnosis, treatment of local recurrence can include salvage chemoradiotherapy, radiation treatment to the area or palliative care (12). In select cases surgical resection may be an option, but this choice will not be appropriate for most patients.

Patients who develop functional dysphagia after esophagectomy may have denervation sustained during the dissection. These patients may suffer from delayed onset of swallow, reduced elevation of the hyoid and decreased opening of the upper esophageal sphincter (13). These problems result in increased risk of aspiration. Improving enteral nutrition by utilizing thickened liquids, as well as training patients to stress protective maneuvers during swallowing, can help to reduce the morbidity from functional dysphagia $(2,13)$. Maintaining enteral nutrition and reducing aspiration risk are key elements in treating functional dysphagia after esophagectomy.

Many patients also report swallowing dysfunction after esophagectomy. Kinematic analyses have shown that there are changes to hyoid movement and location of the epiglottis after esophagectomy that affect swallowing (14). Rehabilitation programs which are instituted early after esophagectomy can alleviate these disorders, however (15).

Several studies have measured the quality of life for patients after esophagectomy. Some studies have reported a worse quality of life for these patients. In particular, patients who underwent a tri-incisional esophagectomy reported more difficulty eating in groups compared to patients who underwent an Ivor-Lewis esophagectomy (16-18).

The location of the anastomosis factors in postoperative quality of life in other ways. A cervical anastomosis may 
be associated with recurrent nerve palsy in 10 percent of cases, while recurrent nerve injury is very rare with an intrathoracic anastomosis. Institutions in the Western Hemisphere are more likely to perform intrathoracic anastomoses, given that more surgeries are done for distal adenocarcinomas. In the Eastern Hemisphere, conversely, more proximal squamous cell carcinomas will require cervical anastomoses to achieve adequate surgical margins.

\section{Gastric conduit}

Dysphagia after esophagectomy can also be secondary to gastric conduit dysfunction. The most common cause of dysfunction is delayed gastric emptying. It is the cause of dysphagia in approximately 30 percent of patients who develop symptoms after esophagectomy (19). Delayed gastric emptying in the first 2-4 weeks after esophagectomy is typical and often a consequence of mucosal swelling. Persistent gastric dysfunction more than 4 weeks after esophagectomy should be evaluated and treated, however. The function of the gastric conduit can be observed via various studies. Barium upper gastrointestinal esophagogram is usually the first test to order including a barium swallow. The esophagogram can identify physical malformations of the conduit and also assess the speed of emptying. In addition, endoscopy will allow for assessment of the structure and integrity of the conduit and opening of the pylorus. CT scan imaging can assess redundancy and level of gastric distension.

Gastric conduit dysfunction can be attributed to dysmotility and altered anatomy of the gastric conduit. Gastric dysmotility inhibits the trajectory of gastric contents and delay gastric emptying, resulting in conduit distention and reflux. Delayed gastric emptying is a common postoperative issue and routinely treated acutely with promotility agents and diet modification in the immediate postoperative setting (20). Typically, as the mucosal swelling subsides the dysphagia resolves. Prolonged dysmotility and delayed emptying, however, can lead to chronic dysphagia and may require additional intervention. Gastric emptying studies allow clinicians to observe the rate at which the stomach accepts and empties food, which can assist in treatment plans. Treatment options includes promotility agents such as erythromycin and metoclopramide. These agents stimulate smooth muscle contraction and increase the speed of gastric emptying (21). Studies also suggest that an implantable gastric nerve stimulator may be effective in intractable cases. The durability of nerve stimulators is unknown, however (22).

A poorly structured gastric conduit can contribute to postoperative dysphagia following esophagectomy. In its normal anatomic position, the stomach uses receptive relaxation to change the pressure in the gastric antrum. Subsequent pyloric relaxation allows for coordinated gastric emptying. The size and shape of the gastric conduit are crucial for its appropriate function when it is mobilized. Given that there is no positive pressure from the intraabdominal cavity to facilitate emptying, the conduit needs to be anatomically upright. Intraoperative gastric tubularization is performed to a width of approximately 3 centimeters. A gastric conduit which is too wide may not empty as efficiently and may become very distended more easily (19). A narrow conduit decreases rates of delayed gastric emptying and acid reflux, which lead to a better quality of life (23). Additionally, there is some evidence that a tubularized gastric conduit may lead to less dysphagia and reflux symptoms (24).

If a patient develops chronic dysphagia postoperatively, an esophagogram can provide information about the shape and position of conduit. Excessive angulation of the conduit can inhibit patency and lead to dysphagia. Similarly, a redundant conduit may develop ineffective peristalsis and require operative intervention. Stricture of the conduit is less common, but may be a mechanism for delayed gastric emptying. Endoscopic balloon dilation may be performed as initial intervention. Dilation or redundancy of the conduit, if extreme, may require surgery to resect the redundant portion. Less than 1 percent of patients who undergo esophagectomy ultimately have surgery performed to revise the conduit (19).

Although most esophagectomies are performed using the posterior mediastinal route, some cases require a substernal location of the gastric conduit. Quality of life studies have not shown significant differences in dysphagia when comparing the posterior mediastinal to the substernal locations (25).

\section{Pylorus}

Postoperative complications due to pyloric dysfunction are reported in up to $15 \%$ of esophagectomy patients due to delayed gastric emptying. These patients present with symptoms of reflux, dysphagia and postprandial pain due to inability of the gastric conduit to become fully decompressed. In debilitated patients, pyloric dysfunction may present with recurrent aspiration and respiratory 
decompensation, with long term risk of malnutrition in already high-risk patients. Dysfunction of the pyloric sphincter occurs as a direct result of vagal destruction during esophagectomy. Upper gastrointestinal swallow will show slow passage of contrast through the pylorus. Endoscopy thereafter will often reveal a tight pylorus, and the scope may not be able to pass into the duodenum. During endoscopy, the scope should be passed through the pylorus if possible to evaluate the integrity of the sphincter, which is difficult to pass in cases of pyloric dysfunction.

During the esophageal mobilization and subsequent resection, the vagus nerve and proximal stomach are transected, leading to changes in the physiologic and neurologic control of the pylorus. Relaxation of the pylorus is caused by inhibitory vagal fibers from the posterior branch of the vagus nerve. The native stomach undergoes receptive relaxation with the intake of food, signaling the antrum and thereafter the pylorus to mobilize gastric contents. Additionally, mobilization of the gastric conduit from the positive pressure in the abdomen to the negative pressure within the thoracic cavity alters the gastric-pyloric pressure differential and limits normal emptying. Resection of the gastric fundus, GE junction, and distal esophagus leads to lack of inhibitory stimulation and ultimately, pyloric spasm.

In order to prevent sequelae of delayed gastric emptying secondary to pyloric denervation, pyloric drainage procedures are often recommended at the time of esophagectomy. A recent meta-analysis showed that the incidence of postoperative gastric outlet obstruction decreased when a pyloric drainage procedure was performed during esophagectomy, though long term outcomes were unchanged (22). With the advancements in minimally invasive esophagectomy, there remains controversy regarding optimal pyloric drainage procedure is ongoing. Pyloromyotomy and pyloroplasty offer definitive division of the pyloric muscle fibers, and are done both minimally invasively and open. The most commonly performed pyloric drainage procedures are the HeinekeMikulicz, Finney, and Jaboulay procedures. Initial analyses showed that pyloromyotomy prevented delayed gastric emptying in over 70 percent of cases (26). However, the addition of pyloric drainage procedure to esophagectomy results in increased operative time, risk of dumping syndrome and enteric leak (7). Some centers perform a Roux-en-Y gastrojejunostomy to facilitate gastric drainage. But the benefit of this additional procedure compared to the increased morbidity of the additional anastomoses is controversial.
Despite multiple retrospective analyses, randomized studies and meta-analyses, there exists no consensus on an optimal pyloric procedure. Studies supporting routine pyloric procedure at time of esophagectomy indicate a lower incidence of delayed gastric emptying and gastric outlet obstruction (27) Additionally, lack of pyloric drainage results in higher rates of aspiration, leak, and complications requiring postoperative intervention (28). However, studies challenging a routine pyloric drainage procedure found no difference in outcomes with and without drainage despite longer operative times (29-31). In a meta-analysis, pyloric drainage resulted in decreased early rates of gastric outlet obstruction, but found no difference in respiratory complications, anastomotic leak, or postoperative mortality (32).

As an alternative to a pyloric drainage procedure, endoscopic techniques can be used during esophagectomy or in the early postoperative period. A variety of endoscopic interventions are described in the literature. Botulinum toxin has proven to be effective for various smooth muscle dysfunction, and it can be injected into the muscle of the pylorus to induce relaxation. Upon visualization of the prepyloric channel, 100-200 international units of botulinum toxin are diluted in saline and are injected equally in four quadrants of the pylorus (33). When compared to pyloroplasty, botulinum toxin injection results in similar rates of delayed gastric emptying but takes less time to perform (34). Botulinum injection can also be performed postoperatively in patients who have developed postoperative dysphagia after esophagectomy. However, botulinum toxin injection is not without risk. Botulinum injection is associated with higher rates of reflux symptoms (35). When compared directly to patients with no intraoperative pyloric intervention, rates of delayed gastric emptying were equivalent in both groups and there was no significant difference in need for additional pyloric procedure (36).

Endoscopic balloon dilation has been described as a successful alternative to botulinum toxin injection for the treatment of pyloric dysfunction. Rates of resolution of gastric outlet obstruction are reported higher than 90 percent, and the complication rate of balloon dilation is low (29). Additionally, pyloric stenting can be utilized in some cases but requires at least one more procedure for eventual retrieval of the stent. Lastly, endoscopic peroral pyloromyotomy is a novel procedure with limited but promising data on outcomes and safety for treatment of gastroparesis (37).

There exists no expert consensus of management of 
the pylorus intraoperatively or postoperatively despite a great deal of available data. Until definitions are widely accepted and additional systematic studies are carried out, surgeons must utilize sound judgment based on the clinical and nutritional status of the patient, difficulty of the operative procedure and an understanding of the operative and nonoperative options to select a strategy for pyloric management.

\section{Diaphragm}

In patients that have completed radiation treatment for esophageal cancer, the hiatal dissection can be challenging, but it is essential to optimize mobility of the conduit in order to maintain a direct course for the passage of contents and minimize postoperative dysphagia. In patients with a large hiatus or extensive hiatal resection, an elevated body mass index (>25 kilograms/meter ${ }^{2}$ ) or a pre-existing hiatal hernia are at increased risk for post-esophagectomy hiatal hernia. Rates of post-esophagectomy hiatal hernia have been reported as high as 19 percent in some studies, but most surgeons have a rate less than 5 percent (36). Hiatal hernia after esophagectomy usually presents in a similar manner to pyloric dysfunction, with dysphagia, nausea, reflux or weight loss. Initial diagnostic studies include upper GI swallow and endoscopy.

Patients that undergo pyloric intervention with endoscopy in the setting of their postoperative dysphagia often have relief of symptoms initially, regardless of etiology. However, in patients with persistent symptoms despite multiple endoscopic interventions, additional imaging should be considered to evaluate the anatomy of the conduit that cannot be appreciated based on endoscopy. CT scan provides this essential information about the course of the conduit and possible extrinsic compression (19).

Hiatal hernia is cited as an uncommon cause of postoperative dysphagia following esophagectomy. Surgeons should note that herniation most commonly occurs into the left chest $(38,39)$. Some surgeons perform routine closure of the crura with a single stitch to narrow the hiatal opening. However, excessive closure of the crura can lead to gastric outlet obstruction and postoperative dysphagia. Fixation of the colon to the subcostal anterior abdominal wall has been suggested to close upper abdominal space where viscera might herniate, but most surgeons do not perform this maneuver (40).

The anatomic shape of the conduit as it passes from the abdomen into the chest can affect gastric emptying.
Tortuosity at the hiatus is due to inadequate mobilization of the proximal duodenum, redundancy or dilation of the conduit leading to distortion in its vertical course through the diaphragm. Surgical revision may be necessary to improve conduit dysfunction in cases of excessive tortuosity. Recommended principles for conduit revision include reducing para-conduit hernias, plication or excision of dilated conduit, straightening of an angled conduit and gastric drainage with or without pyloric intervention (19).

Dysphagia due to obstruction by a tight hiatus can be difficult to diagnose due to the high frequency of postoperative pyloric dysfunction and anatomic location of the pylorus after esophagectomy. A high index of suspicion is essential to early diagnosis and appropriate intervention. Especially in cases of para-conduit hernia, morbidity is high and increases with delay in diagnosis.

\section{Conclusions}

To perform esophagectomy successfully, surgeons must understand relevant anatomy and also be committed to managing symptoms and complications on a long-term basis. Following esophagectomy, dysphagia is a complex and potentially morbid complication which should be evaluated systematically. Utilizing an algorithm based on available data and recommendations will evaluate the four anatomic areas most commonly associated with postoperative dysphagia.

\section{Acknowledgments}

Funding: None.

\section{Footnote}

Reporting Checklist: The authors have completed the Narrative Review reporting checklist. Available at https:// dx.doi.org/10.21037/jtd-21-724

Peer Review File: Available at https://dx.doi.org/10.21037/ jtd-21-724

Conflicts of Interest: All authors have completed the ICMJE uniform disclosure form (available at https://dx.doi. org/10.21037/jtd-21-724). IO serves as an unpaid editorial board member of Fournal of Thoracic Disease from Feb 2021 to Jan 2023. The authors have no other conflicts of interest to declare. 
Ethical Statement: The authors are accountable for all aspects of the work in ensuring that questions related to the accuracy or integrity of any part of the work are appropriately investigated and resolved.

Open Access Statement: This is an Open Access article distributed in accordance with the Creative Commons Attribution-NonCommercial-NoDerivs 4.0 International License (CC BY-NC-ND 4.0), which permits the noncommercial replication and distribution of the article with the strict proviso that no changes or edits are made and the original work is properly cited (including links to both the formal publication through the relevant DOI and the license). See: https://creativecommons.org/licenses/by-nc-nd/4.0/.

\section{References}

1. Park JY, Song HY, Kim JH, et al. Benign anastomotic strictures after esophagectomy: long-term effectiveness of balloon dilation and factors affecting recurrence in 155 patients. AJR Am J Roentgenol 2012;198:1208-13.

2. Chen KN. Managing complications I: leaks, strictures, emptying, reflux, chylothorax. J Thorac Dis 2014;6 Suppl 3:S355-63.

3. Lou F, Sima CS, Adusumilli PS, et al. Esophageal cancer recurrence patterns and implications for surveillance. J Thorac Oncol 2013;8:1558-62.

4. Tabatabai A, Hashemi M, Mohajeri G, et al. Incidence and risk factors predisposing anastomotic leak after transhiatal esophagectomy. Ann Thorac Med 2009;4:197-200.

5. Price TN, Nichols FC, Harmsen WS, et al. A comprehensive review of anastomotic technique in 432 esophagectomies. Ann Thorac Surg 2013;95:1154-60; discussion 1160-1.

6. Koëter M, van der Sangen MJ, Hurkmans CW, et al. Radiation dose does not influence anastomotic complications in patients with esophageal cancer treated with neoadjuvant chemoradiation and transhiatal esophagectomy. Radiat Oncol 2015;10:59.

7. Kim JH, Shin JH, Song HY. Benign strictures of the esophagus and gastric outlet: interventional management. Korean J Radiol 2010;11:497-506.

8. Kim HC, Shin JH, Song HY, et al. Fluoroscopically guided balloon dilation for benign anastomotic stricture after Ivor-Lewis esophagectomy: experience in 62 patients. J Vasc Interv Radiol 2005;16:1699-704.

9. Blackmon SH, Correa AM, Wynn B, et al. Propensitymatched analysis of three techniques for intrathoracic esophagogastric anastomosis. Ann Thorac Surg 2007;83:1805-13; discussion 1813.

10. Dasari CS, Jegadeesan R, Patel HK, et al. Intralesional steroids and endoscopic dilation for anastomotic strictures after esophagectomy: systematic review and meta-analysis. Endoscopy 2020;52:721-6.

11. Price TN, Allen MS, Nichols FC 3rd, et al. Hiatal hernia after esophagectomy: analysis of 2,182 esophagectomies from a single institution. Ann Thorac Surg 2011;92:2041-5.

12. Ni W, Yang J, Deng W, et al. Patterns of recurrence after surgery and efficacy of salvage therapy after recurrence in patients with thoracic esophageal squamous cell carcinoma. BMC Cancer 2020;20:144.

13. Kaneoka A, Yang S, Inokuchi H, et al. Presentation of oropharyngeal dysphagia and rehabilitative intervention following esophagectomy: a systematic review. Dis Esophagus 2018;31:doy050.

14. Kim SJ, Cheon HJ, Lee HN, et al. Kinematic analysis of swallowing in the patients with esophagectomy for esophageal cancer. J Electromyogr Kinesiol 2016;28:208-13.

15. Takatsu J, Higaki E, Hosoi T, et al. Clinical benefits of a swallowing intervention for esophageal cancer patients after esophagectomy. Dis Esophagus 2021;34:doaa094.

16. Jezerskyte E, Saadeh LM, Hagens ERC, et al. Longterm health-related quality of life after McKeown and Ivor Lewis esophagectomy for esophageal carcinoma. Dis Esophagus 2020;33:doaa022.

17. Yang YS, Shang QX, Yuan Y, et al. Comparison of Longterm Quality of Life in Patients with Esophageal Cancer after Ivor-Lewis, Mckeown, or Sweet Esophagectomy. J Gastrointest Surg 2019;23:225-31.

18. Ha JS, Battafarano RJ. Complications of Esophageal Resection. In: Baumgartner WA, Darling GE, Jacobs JP. editors. STS Cardiothoracic Surgery E-Book. Chicago: Society of Thoracic Surgeons, 2020.

19. Rove JY, Krupnick AS, Baciewicz FA, et al. Gastric conduit revision postesophagectomy: Management for a rare complication. J Thorac Cardiovasc Surg 2017;154:1450-8.

20. Lacy BE, Weiser K, Kennedy A. Botulinum toxin and gastrointestinal tract disorders: panacea, placebo, or pathway to the future? Gastroenterol Hepatol (N Y) 2008;4:283-95.

21. Burt M, Scott A, Williard WC, et al. Erythromycin stimulates gastric emptying after esophagectomy with gastric replacement: a randomized clinical trial. J Thorac Cardiovasc Surg 1996;111:649-54.

22. Asti E, Lovece A, Bonavina L. Thoracoscopic Implant of 
Neurostimulator for Delayed Gastric Conduit Emptying After Esophagectomy. J Laparoendosc Adv Surg Tech A 2016;26:299-301.

23. Akkerman RD, Haverkamp L, van Hillegersberg R, et al. Surgical techniques to prevent delayed gastric emptying after esophagectomy with gastric interposition: a systematic review. Ann Thorac Surg 2014;98:1512-9.

24. Castle SL, Isani M, Torres MB, et al. Tubularized Gastric Conduit is More Desirable in Pediatric Patients Treated with Minimally Invasive Esophagectomy and Gastric PullUp. J Laparoendosc Adv Surg Tech A 2017;27:427-9.

25. Urschel JD, Blewett CJ, Young JE, et al. Pyloric drainage (pyloroplasty) or no drainage in gastric reconstruction after esophagectomy: a meta-analysis of randomized controlled trials. Dig Surg 2002;19:160-4.

26. Kim D. The Optimal Pyloric Procedure: A Collective Review. Korean J Thorac Cardiovasc Surg 2020;53:233-41.

27. Fok M, Cheng SW, Wong J. Pyloroplasty versus no drainage in gastric replacement of the esophagus. Am J Surg 1991;162:447-52.

28. Antonoff MB, Puri V, Meyers BF, et al. Comparison of pyloric intervention strategies at the time of esophagectomy: is more better? Ann Thorac Surg 2014;97:1950-7; discussion 1657-8.

29. Lanuti M, DeDelva P, Morse CR, et al. Management of delayed gastric emptying after esophagectomy with endoscopic balloon dilatation of the pylorus. Ann Thorac Surg 2011;91:1019-24.

30. Cerfolio RJ, Bryant AS, Canon CL, et al. Is botulinum toxin injection of the pylorus during Ivor Lewis corrected esophagogastrectomy the optimal drainage strategy? J Thorac Cardiovasc Surg 2009;137:565-72.

31. Gaur P, Swanson SJ. Should we continue to drain the pylorus in patients undergoing an esophagectomy? Dis

Cite this article as: Benz C, Martella J, Hamwi B, Okereke I. Factors resulting in postoperative dysphagia following esophagectomy: a narrative review. J Thorac Dis 2021;13(7):4511-4518. doi: 10.21037/jtd-21-724
Esophagus 2014;27:568-73.

32. Khan OA, Manners J, Rengarajan A, et al. Does pyloroplasty following esophagectomy improve early clinical outcomes? Interact Cardiovasc Thorac Surg 2007;6:247-50.

33. Bard V. Delayed Gastric Emptying Following Transhiatal Esophagectomy without Pyloroplasty: Our Experience with Endoscopic Botox Injection. MOJ Clin Med Case Rep 2016;4(6).

34. Bagheri R, Fattahi SH, Haghi SZ, et al. Botulinum toxin for prevention of delayed gastric emptying after esophagectomy. Asian Cardiovasc Thorac Ann 2013;21:689-92.

35. Eldaif SM, Lee R, Adams KN, et al. Intrapyloric botulinum injection increases postoperative esophagectomy complications. Ann Thorac Surg 2014;97:1959-64; discussion 1964-5.

36. Stewart CL, Wilson L, Hamm A, et al. Is Chemical Pyloroplasty Necessary for Minimally Invasive Esophagectomy? Ann Surg Oncol 2017;24:1414-8.

37. Mekaroonkamol P, Shah R, Cai Q. Outcomes of per oral endoscopic pyloromyotomy in gastroparesis worldwide. World J Gastroenterol 2019;25:909-22.

38. M Hennessy M, Ivanovski I, Spartalis E, et al. Diaphragmatic hernia following esophagectomy for esophageal cancer: A systematic review. J BUON 2019;24:1793-800.

39. Vallböhmer D, Hölscher AH, Herbold T, et al. Diaphragmatic hernia after conventional or laparoscopicassisted transthoracic esophagectomy. Ann Thorac Surg 2007;84:1847-52.

40. Bronson NW, Luna RA, Hunter JG, et al. The incidence of hiatal hernia after minimally invasive esophagectomy. J Gastrointest Surg 2014;18:889-93. 